\title{
Contributions of the microbiome to intestinal inflammation in a gut-on-a-chip
}

\author{
Min Seo Jeon ${ }^{1 \dagger}$, Yoon Young $\mathrm{Choi}^{2 \dagger}$, Sung Jun $\mathrm{Mo}^{3}$, Jang Ho Ha ${ }^{4}$, Young Seo Lee ${ }^{4}$, Hee Uk Lee ${ }^{4}$, \\ Soo Dong Park ${ }^{3}$, Jae-Jung Shim ${ }^{3}$, Jung-Lyoul Lee ${ }^{3}$ and Bong Geun Chung ${ }^{4^{*}}$ (D)
}

\begin{abstract}
The intestinal microbiome affects a number of biological functions of the organism. Although the animal model is a powerful tool to study the relationship between the host and microbe, a physiologically relevant in vitro human intestinal system has still unmet needs. Thus, the establishment of an in vitro living cell-based system of the intestine that can mimic the mechanical, structural, absorptive, transport and pathophysiological properties of the human intestinal environment along with its commensal bacterial strains can promote pharmaceutical development and potentially replace animal testing. In this paper, we present a microfluidic-based gut model which allows co-culture of human and microbial cells to mimic the gastrointestinal structure. The gut microenvironment is recreated by flowing fluid at a low rate $(21 \mu \mathrm{L} / \mathrm{h})$ over the microchannels. Under these conditions, we demonstrated the capability of gut-on-a-chip to recapitulate in vivo relevance epithelial cell differentiation including highly polarized epithelium, mucus secretion, and tight membrane integrity. Additionally, we observed that the co-culture of damaged epithelial layer with the probiotics resulted in a substantial responded recovery of barrier function without bacterial overgrowth in a gut-ona-chip. Therefore, this gut-on-a-chip could promote explorations interaction with host between microbe and provide the insights into questions of fundamental research linking the intestinal microbiome to human health and disease.
\end{abstract}

Keywords: Gut-on-a-chip, Inflammation bowel disease, Microbiome, Shear stress

\section{Introduction}

The intestine is a primary organ for digestion, absorption, and metabolism of the nutrients and drugs, establishing a protective barrier between the pathogen and other harmful microorganisms in a human body [1-3]. Furthermore, the intestine is the major site for cross-talk between the intestinal epithelium and commensal microbes of the gut microbiome $[4,5]$. In general, the gut microbiome significantly contributes toward protection of the hosts against pathogenic incursions by enhancing the host defense mechanism [6]. For this reason, an imbalance between the composition and function of the intestinal microbes is associated with a number of diseases, such

\footnotetext{
*Correspondence: bchung@sogang.ac.kr

${ }^{\dagger}$ Min Seo Jeon and Yoon Young Choi have equally contributed to this work

${ }^{4}$ Department of Mechanical Engineering, Sogang University, Seoul, Korea Full list of author information is available at the end of the article
}

as inflammatory bowel disease (IBD), diabetes, obesity, cancer, and neurodegenerative diseases [7-9]. Thus, the human microbiome is appearing as a key player governing human health and the investigation of host-microbiome interaction emerges the substantial significance for drug development and disease treatment.

To study the interaction between gut microbiomes and host cells, there have been great efforts to develop experimental in vitro and in vivo models of the intestinal system that can be used to analyze intestinal physiology both in the present and absence of living gut microbiomes [10]. Over the past decades, the mimicking of the intestinal microenvironment has extensively been studied. The mammalian animal models $[11,12]$ and static transwell models $[13,14]$ are most commonly utilized to mimic the human intestinal system for investigations of human intestinal physiology and the microbiome. However, the animal model experiment is expensive, concerned about 
ethical and legal frameworks, and not fully scalable to human physiological responses. The experimental evidence showed that the static transwell models did not recapitulate the relevant aspects of the complex cellular diversity and dynamics (e.g., morphophysiology) of the human intestine, resulting in poorly reflection of in vivo physiology $[15,16]$. Furthermore, the static transwell culture requires almost 3 weeks for the intestinal epithelial cells to differentiate into the intestinal lineage cells and fails to reveal some key intestinal differentiated functions $[17,18]$. Recently, a three-dimensional (3D) human tissue surrogate, such as intestinal organoid (known as enteroid), has been emerged as a promising alternative due to its limitation of physiological relevance between living human intestinal system with intestinal cell lines [19-21]. However, one of the major drawbacks of the enteroids is the absence of mechanically active microenvironment of the living intestine (e.g., peristaltic motions or intralumenal fluidic flow) [22] that is critical for normal organ physiology as well as for development of Crohn's disease and other intestinal disorders. Additionally, most of these in vitro intestinal models have not been adaptable to grow living microbes on the luminal surface of cultured intestinal epithelium due to overgrowth of microbes [23, $24]$, which usually dominate the co-culture system and induce human cell apoptosis within a day. This is a key problem, because the microbial symbionts normally contribute to intestinal barrier function, metabolism, and absorption of drugs and chemicals.

Recently, the development of the microfluidic technology has suggested an effective way to emulate the human intestinal environment, typically called "guton-a-chip". This microfluidic platform includes controllable multiple system parameters (e.g., fluidic flow and oxygen concentration, mechanical deformation) to recapitulate the intestinal microenvironment situation. A number of researchers have developed gut-on-chip systems that could emulate intestinal environment to improve the cellular differentiation of human intestinal epithelial cells (Caco-2) and investigate the between the microbiome and host cells [25-27]. In most cases, the viability of the intestinal epithelium was better than transwell insert systems, thus allowing the long-term co-culture of microbiomes with host cells. However, one of the major limitations is that it relies on a complex device mechanically stretch intestinal epithelial cells rather than periodically wriggle like in vivo intestinal system. Moreover, the most of these studies were performed in the absence of other supporting cells and tissue types found within in vivo intestine system (e.g., blood vessel), which is important for disease modeling $[28,29]$. Here, we developed the human gut-on-a-chip model that could emulate some relevant features and dynamic behaviors of the human intestinal tract that would overcome these limitations. First, we designed a three-channel microfluidic-based gut-on-a-chip to enable the co-culture Caco-2 cells with other supporting cells in the presence of physiologically relevant intestinal luminal flow, which could stimulate the differentiation of intestinal epithelial cell lineages of the small intestine, as previously described [30,31]. Second, the osmotic pump was used to create flow in lumen fluids of the intestine and vessel, mimicking the stable fluidic flow of the intestinal lumen in vivo. We further analyzed the differentiation, microvilli, glycocalyx layer secretion, and barrier functions of the intestinal epithelial cells in a human gut on-chip. We also established a disease model of lipopolysaccharide (LPS)-induced intestinal injury and inflammation and assessed therapeutic effects of probiotics.

\section{Materials and methods}

\subsection{Fabrication of the gut-on-a-chip with microelectrode arrays}

The microfluidic-based gut-on-a-chip with microelectrode arrays was designed using Autocad (Autodesk, California, USA). The gut-on-a-chip master mold was made with SU-8 photoresist (MicroChem Corp., Massachusetts, USA) on a silicon wafer. SU-8 100 photoresist was spin-coated with $2000 \mathrm{rpm}$ for $30 \mathrm{~s}$ on a 4-inch silicon wafer and baked at $65{ }^{\circ} \mathrm{C}$ for $20 \mathrm{~min}, 95{ }^{\circ} \mathrm{C}$ for $1 \mathrm{~h}$, respectively. After soft baking, an UV light was exposed for $40 \mathrm{~s}$ with UV aligner (MDA-400LJ, Midas System Co. Ltd, Daejeon, Korea) and developed unexposed photoresist for $12 \mathrm{~min}$. Microelectrode arrays were fabricated by E-beam physical vapor deposition. A positive photoresist was deposited on transparent soda-lime glass wafer (iNexus Inc., Gyeonggi, Korea) and developed by exposure to UV light using a mask aligner (Suss MA6 Mask Aligner, SUSS MicroTec AG, Garching, Germany). The chromium was sprayed onto the glass wafer to make a $5 \mathrm{~nm}$ thickness using an E-beam evaporator (ULVAC Inc., Kanagawa, Japan) and gold was then sprayed to have a $50 \mathrm{~nm}$ height. After sputtering, the unexposed portion of the photoresist was removed to form a metal electrode pattern. The polydimethylsiloxane (PDMS)-based gut-on-a-chip mold was prepared using a 10:1 mixture of a silicone elastomer and curing agent (Sylgard 184, Dow Corning Corp., Michigan, USA). The air bubbles were removed by vacuum for $30 \mathrm{~min}$ and PDMS-based gut-on-a-chip was polymerized at $80{ }^{\circ} \mathrm{C}$ for $1 \mathrm{~h}$. The gut-on-a-chip mold were treated with in a plasma cleaner (Femto Science, Gyeonggi, Korea) to bond with microelectrode array substrates. 


\subsection{Osmosis-driven fluidic flow set-up}

PDMS cubic chambers with one cellulose membrane window were fabricated to make the osmotic pump using conventional protocols, as previously described [32]. The bonding of a cellulose membrane with a PDMS chamber was performed using the PDMS solution as adhesive glue. As a preliminary study, we conducted the osmosis experiments to evaluate the pumping capability of the osmotic pump. The deionized water was used as a buffer solution and polyethylene glycol (PEG) (Sigma-Aldrich, MO, USA; 2000 molecular weight) solution was used as a driving agent.

\subsection{Computational fluid dynamics (CFD) simulation}

The microchannel modeling was performed with Autodesk Inventor (Autodesk Inc. USA) 3D CAD software and imported to COMSOL Multiphysics 5.5 (Comsol Inc., USA) software to conduct fluidic flow modeling. The fluidic fluid was assumed to be water and the microchannel wall settings were set to no slip condition. The extracellular matrix (ECM) channel was modeled using the physical properties of Collagen type I (Gibco ${ }^{\mathrm{TM}}$, Thermo Fisher Scientific, USA) with a density of $0.8536 \mathrm{~kg} / \mathrm{m}^{3}$ and a viscosity of $6.6104 \mathrm{~Pa} \cdot \mathrm{s}$. The hexagonal-shaped micropillars with a width of $500 \mu \mathrm{m}$ and a thickness of $150 \mu \mathrm{m}$ were placed in a straight line between the microchannels. The height and width of the epithelial cell channel were defined in the range of $50 \mu \mathrm{m}$ to $250 \mu \mathrm{m}$ and $600 \mu \mathrm{m}$ to $1000 \mu \mathrm{m}$, respectively, to determine the effect of channel dimensions on the fluid flow velocity profile and the wall shear stress acting between two channels. We calculated the fluid flow velocity profile by assuming the steady-state, singlephased laminar flow (SPF) with the inflow rates set to $0.5,2.0,3.5,5.0$, and $6.5 \mu \mathrm{L} / \mathrm{min}$, respectively. Furthermore, the wall shear stress acting on the cell surface was calculated with the equation $\tau_{\mathrm{w}}=\mu \bullet \dot{\gamma}$, where $\mu$ is the dynamic viscosity and $\dot{\gamma}$ is the shear rate (at the boundary of the cell surface). In our gut-on-a-chip system, the fluid flow through the microchannels was induced by osmotic pump. The flow rate is determined by the molar concentration of the PEG (Sigma-Aldrich, USA) solution. The average transferred volume was approximately $3.5 \mu \mathrm{L} / \mathrm{min}$ for a $0.18 \mathrm{M}$ PEG solution, $7.0 \mu \mathrm{L} / \mathrm{min}$ for a $0.36 \mathrm{M}$ PEG solution, and $10.5 \mu \mathrm{L} / \mathrm{min}$ for a $0.72 \mathrm{M}$ PEG solution, respectively. To investigate the fluid velocity distribution inside the microchannel, we calculated the velocity in A-D sections of the central microchannel (Fig. 2D). The simulations were performed in the same method of the preceding conditions with respect to the molecular concentrations.

\subsection{Preparation and cell seeding on a gut-on-a-chip}

The gut-on-a-chip was sterilized by autoclaving $\left(120{ }^{\circ} \mathrm{C}\right.$ for $30 \mathrm{~min}$ ) and were dried in an oven. The gut-on-achips were coated with $1 \mathrm{mg} / \mathrm{mL}$ Poly-D-lysine (Sigma Aldrich, MO, USA) for overnight to improve cell adhesion and prevent the detachment of collagen type I gels. After coating, the gut-on-a-chip was rinsed with a deionized water more than three times, then placed in a dish at $80{ }^{\circ} \mathrm{C}$ in an oven at least $24 \mathrm{~h}$. The collagen type I gel (Invitrogen, USA) with $2 \mathrm{mg} / \mathrm{mL}$ density was gently and slowly filled into the central channel. We placed the collagen gel-filled gut-on-a-chip in a $\mathrm{CO}_{2}$ incubator for $30 \mathrm{~min}$ to allow the collagen to gel. When the collagen solution was completely to gel, secondary coating was performed to epithelial cell channel with collagen and matrigel mixture for 15 min before seeding the Caco-2 cells. The human Caco-2 cells (ATCC clone HTB-37) were cultured in a modified Eagle's medium with $10 \%$ fetal bovine serum, nonessential amino acids, L-glutamin, and penicillin-streptomycin in the absence of Calcium. The human umbilical vein endothelial cells (HUVECs) were cultured in EBM-2 basal medium with supplements and growth factors. Caco- 2 cell and HUVEC suspension $\left(100 \mu \mathrm{L}, 1 \times 10^{4}\right.$ cells/ $\left.\mathrm{mL}\right)$ were loaded into the microchannel using a micropipette. The cells in the suspension medium flowed into the microchannel by a gravity and were spontaneously trapped in the microchannel. As the cell suspension with homogenous density was applied in microchannel, the regular amounts of cells were allocated in each microchannel. We left the cells in the incubator for overnight without any treatment for stabilization of cells within the microchannel. After the cells were attached to the microchannel, the non-adherent cells were washed out. The outlet was connected with the flexible polyurethane tube.

\subsection{Immunofluorescence staining}

For immunofluorescence microscopic analysis, the cells grown in the gut-on-a-chip were fixed with $4 \%$ (wt/vol) paraformaldehyde for $15 \mathrm{~min}$, washed twice for $5 \mathrm{~min}$ with $0.1 \%$ bovine serum albumin (BSA) in phosphate buffer saline (PBS), and then permeabilized with $0.2 \%$ (vol/vol) Triton X-100 (Sigma Aldrich, MO, USA) for $20 \mathrm{~min}$. After washing with $0.1 \%$ BSA in PBS, the cells were incubated with $3 \%$ (wt $/ \mathrm{vol}$ ) BSA blocking solution for $1 \mathrm{~h}$. Subsequently, the cells were incubated with primary antibodies overnight at $4{ }^{\circ} \mathrm{C}$, washed three times, incubated with secondary antibodies for $90 \mathrm{~min}$, and washed three times with $0.1 \%$ BSA in PBS. The following antibodies were used for immunohistochemistry: mouse anti-ZO-1 (Invitrogen, USA, 1:200), rabbit anti-PECAM 
(Abcam, 1:500), mouse anti-MUC2 (Invitrogen, USA, 1:500), Alexa Fluor 594-conjugated phalloidin (Invitrogen, USA, 1:250), Alexa Fluor 488-conjugated Wheat Germ Agglutinin (Invitrogen, USA, $5 \mu \mathrm{g} / \mathrm{mL}, 1: 200$ ), Goat anti-mouse Alexa Fluor 488 (Invitrogen, USA, 1:1000), and Donkey anti-rabbit Alexa Fluor 594 (Invitrogen, USA, 1:1000). Samples were then incubated with 4',6-diamidino-2-phenylindole dihydrochloride (DAPI; Molecular Probe, OR, USA) to visualize cell nuclei before taking confocal microscopic images (Olympus, Japan).

\subsection{Bacterial cell culture and live staining}

Lactiplantibacillus plantarum HY7715 (HY7715) probiotic and Bifidobacterium animalis spp. lactis HY8002 (HY8002) probiotic were supplied by hy Co., Ltd. (Yongin-si, Korea). Lactiplantibacillus plantarum type strain ATCC14917 (ATCC14917) from the American Type Culture Collection (ATCC; Manassas, VA, USA) was used a reference strain to evaluate intestinal stability. $L p b$. plantarum strains were grown in Man, Rogosa and Sharp (MRS) broth (BD, Franklin Lakes, NJ, USA) and $B$. animalis spp. lactis strain was grown in BL broth (MBcell, Seoul, Korea) at $37{ }^{\circ} \mathrm{C}$ for $24 \mathrm{~h}$. Subsequently, the bacterial cells were harvested by centrifugation $(4,000 \mathrm{rpm}$, $10 \mathrm{~min}, 4^{\circ} \mathrm{C}$ ), washed three times with PBS, and resuspended in cell culture media at $10^{9} \mathrm{CFU} / \mathrm{mL}$ before each assay. When bacterial cells were harvested by centrifugation (5000 g, $15 \mathrm{~min}$ ) the cells were resuspended with $2 \mathrm{~mL}$ of raw EMEM. After suspension, the dye mixture of equal volumes ( $3 \mu \mathrm{L}$ to each milliliter) of $\mathrm{SYTO}^{\circledR} 9$ and propidium iodide (LIVE/DEAD ${ }^{\circledR}$ BacLight $^{\mathrm{TM}}$ Bacterial Viability Kit, L7012, Thermo Fisher Scientific) was added. The cells with dye mixtures were incubated at room temperature in the dark for $15 \mathrm{~min}$. After the two-time centrifugation and washing, they were inoculated in the Caco-2 cell microchannel.

\subsection{Inflammation study}

To mimic the chronically inflamed microenvironment with engineered intestinal villi grown in a gut-on-a-chip was administrated the $15 \mu \mathrm{g} / \mathrm{mL}$ of lipopolysaccharide (LPS) (Invitrogen, USA) to the epithelial channel for $24 \mathrm{~h}$. The ready to use eBioscience ${ }^{\mathrm{TM}}$ LPS (from Escherichia coli 026: B6) solution was directly diluted in raw EMEM to give an appropriate final concentration. Briefly, 5 days co-cultured intestinal models were treated $15 \mu \mathrm{g} /$ $\mathrm{mL}$ of LPS concentrations for $24 \mathrm{~h}$. After the inflammation induction, the pre-cultivated HY7715 probiotic was resuspended in a culture medium and cultivated in LPSinduced barrier dysfunction model at $37{ }^{\circ} \mathrm{C}$ for 3 days under the fluidic culture condition. After cell density was adjusted to $1 \times 10^{-8} \mathrm{CFU} / \mathrm{mL}$, HY7715 probiotic was introduced into the epithelium channel of the cocultured model.

\subsection{Epithelial barrier analysis}

The impedance was recoded using an alternating current (AC) voltage signal with an amplitude of $10 \mathrm{mV}$ at a frequency range of $10 \sim 100 \mathrm{kHz}(\mathrm{CHI} 660 \mathrm{E}$, electrochemical workstation). The transepithelial electrical resistance (TEER) was obtained by determining a suitable readout frequency from the impedance. TEER values are typically reported in units of $\Omega \cdot \mathrm{cm}^{2}$ and calculated as: TEER $=R$ $(\Omega) \times \mathrm{M}_{\text {area }}\left(\mathrm{cm}^{2}\right)$, as previously described $[33,34]$ where, $R$ is the cell-specific resistance across the cell layer, $M_{\text {area }}$ is the effective area $\left(0.0028 \mathrm{~cm}^{2}\right)$. The magnitude of the impedances for these two electrode pairs were averaged for each session as average of $|\mathrm{Z}|$. To obtain solely the information attributed to the growing cell layer, the magnitude of the impedances for each electrode pair of the measurements prior to cell seeding was subtracted from all the subsequent measurements during cell culture, resulting in the relative magnitude of the impedance [35].

\subsection{Statistical analysis}

All results and error bars are represented as mean \pm SEM. Statistical significance of differences between mean values was assessed with t-tests for unpaired data (GraphPad Prism software version 8.00 for Windows; GraphPad Software, Inc., San Diego, CA). A two-tailed unpaired student's t-test was used to test significance between individual data sets as indicated. $p$-value less than 0.05 was considered statistically significant. All experiments were repeated at least three times to ensure reproducibility.

\section{Result and discussion}

\subsection{Fabrication of a human gut on-chip model and CFD simulation analysis}

To investigate the interactions between microbiomes and human Caco-2 cells in a human intestinal microenvironment, we developed a microfluidic-based gut-on-a-chip embedding with microelectrode arrays (Fig. 1A). The gut-on-a-chip consisted of three parallel microchannels $(500 \mu \mathrm{m}$ wide, $10 \mathrm{~mm}$ long, $150 \mu \mathrm{m}$ high) separated by the hexagonal-shaped micropillars: two stromal cell culture channels which could mimic the epithelium or endothelium layer and one central channel filled with collagen type I gel (Fig. 1A, right). We used the three parallel microchannels separated by collagen gel channel without any polymeric membrane, showing that it was relatively easy to fabricate. By using an osmotic pump, the culture medium was perfused at simulated flow rates through each microchannel, mimicking the fluidic flow and associated shear stress on the cell surface in the human 


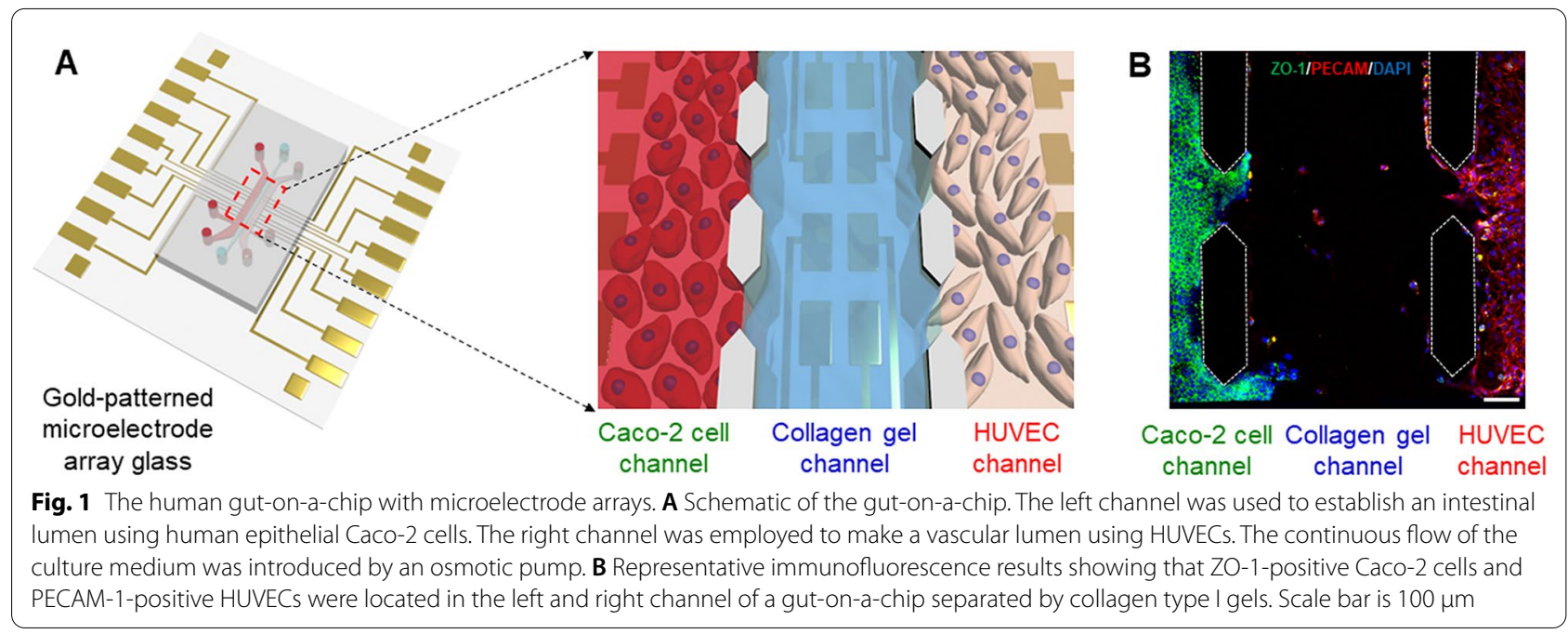

intestinal lumen and the blood vessels in vivo. We cocultured Caco- 2 cells and HUVECs separated by collagen gels in a gut-on-a-chip (Fig. 1B). Prior to the experiment, the flow dynamics of the gut-on-a chip were performed in CFD simulation to assess the correlation between wall shear stress and the dimensions of the microfluidic channel (Fig. 2). Within the microchannel, the flow velocity $u$ is dominated by the homogeneous, incompressible Naiver-Stokes equation and continuity equation:

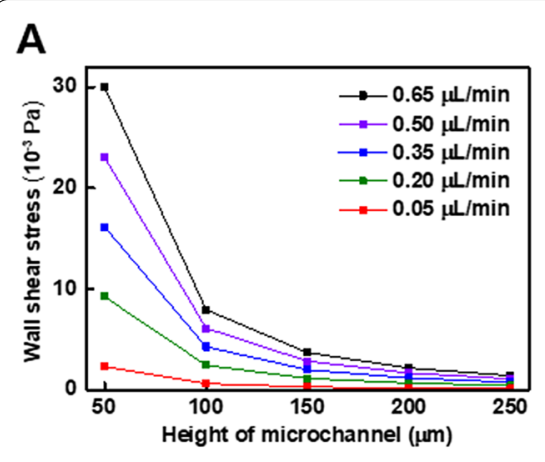

D

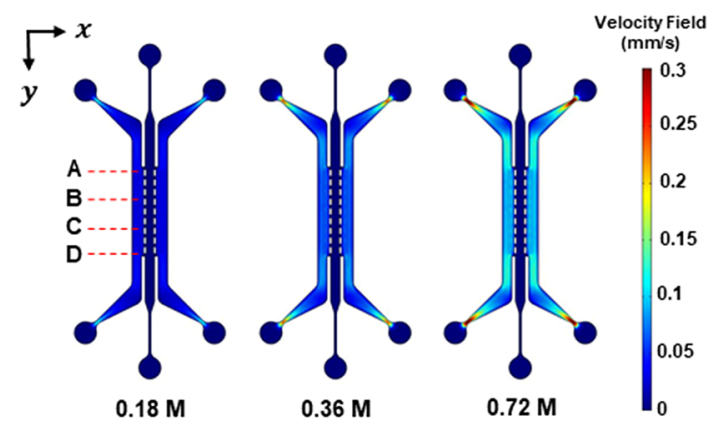

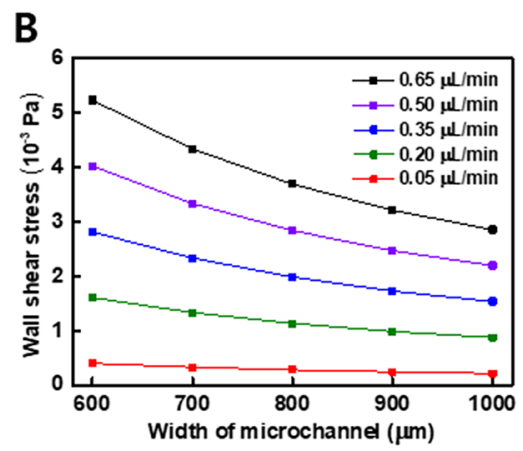

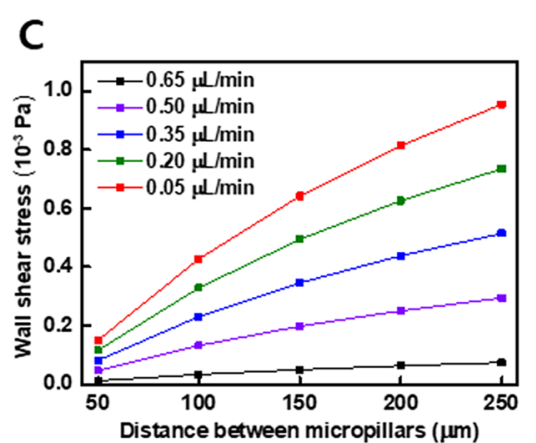

E

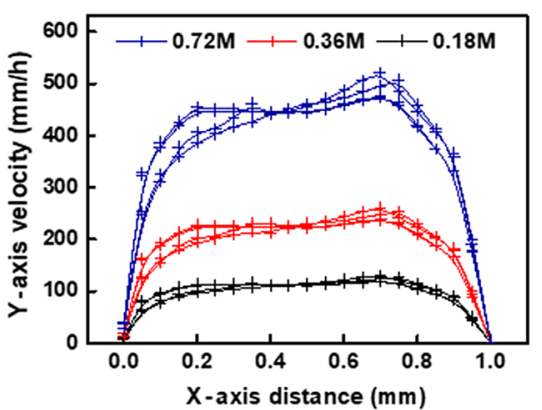

Fig. 2 Simulation of a gut-on-a-chip under various fluidic flows and geometric conditions. A Wall shear stress is calculated with respect to the height of the microchannel. B Wall shear stress is calculated with respect to the width of the microchannel. $\mathbf{C}$ Wall shear stress is calculated with respect to the distance between the micropillars. D Flow speed distribution in the cell culture microchannel. CFD simulations were performed to analyze the velocity at various concentrations of $0.18 \mathrm{M}, 0.36 \mathrm{M}, 0.72 \mathrm{M}$ PEG solutions. E Velocity distribution inside the microchannel is calculated in a section of $\mathbf{A}-\mathbf{D}$ 


$$
\begin{aligned}
& \rho(u \bullet \nabla) u=\nabla \bullet[-p \mathrm{I}+\tau]+F \\
& \rho \nabla \bullet u=0
\end{aligned}
$$

where $\rho$ is the fluid density, $u$ is the velocity field, $\nabla$ is the divergence, $p$ is the pressure, $\tau$ is the Stoke's stress $\left(\tau=\mu\left(\nabla u+\nabla u^{T}\right)\right), \mu$ is the dynamic viscosity, and $F$ is the volume force. In consequence, the wall shear stress acting on the epithelial cell channel was derived from the previously obtained velocity field. Assuming an isotropic Newtonian flow in the channel, the shear stress inside the shear stress inside the microfluidics can be calculated by the following equation:

$$
\tau(\vec{u})=\mu \nabla \vec{u}
$$

where $\mu$ is the dynamic viscosity, and $\nabla \vec{u}$ is the gradient of velocity field which is also called as wall shear rate. The average value of the wall shear stress was acquired from the boundary of the epithelial cell channel in which the lactic acid bacteria were cultured. The height and width of epithelial cell channel and the distance between the micropillars are set as parameters. The dimensions of the parameters were set in the ranges of 50 to $250 \mu \mathrm{m}, 600$ to $1000 \mu \mathrm{m}$, and 50 to $250 \mu \mathrm{m}$, respectively. It is important to find the optimal microchannel dimension for the well growth of lactic acid bacteria. Since the wall shear stress acting at the boundary surface is a crucial factor for the cell growth [36], the correlation between channel dimension and wall shear stress needs to be considered to determine optimal channel dimension. Simulating with respect to the microchannel height, the wall shear stress rapidly decreased as the channel height was increased (Fig. 2A). The average wall shear stress values for the channel height of $0.05,0.2,0.35,0.5$, and $0.65 \mu \mathrm{m}$ at a flow rate of $0.35 \mu \mathrm{L} / \mathrm{min}$ were $16.16,4.28$, $1.99,1.17$, and $0.7610^{-3} \mathrm{~Pa}$, respectively. The lower the height of microchannels, the higher the wall shear stress. From the results of CFD simulation for the microchannel width, the wall shear stress was proportional to the microchannel width (Fig. 2B). Within a fixed flow rate, the changes in microchannel height and width leaded to variation in channel volume, which could affect the flow velocity. Simulating with respect to micropillar distance, the wall shear stress was increased with the micropillar distance (Fig. 2C). The flow rate was simulated in the range between 0.05 and $0.65 \mu \mathrm{L} / \mathrm{min}$ for each parameter. All three simulations showed the same tendency for wall shear stress to increase in proportion to the flow rate, because the laminar flow shared the same streamline in the identical geometry [37]. The distribution of the flow velocity in the channel at the different concentrations of PEG solutions were calculated (Fig. 2D). In the case of flow driven by $0.18 \mathrm{M}$ PEG solution, the velocity was relatively uniform over most of the channel, whereas the flow with $0.36 \mathrm{M}$ and $0.72 \mathrm{M}$ PEG solutions exhibited wide variations between the upper and lower flows. The velocity at a position of $\mathrm{A}, \mathrm{B}, \mathrm{C}$, and $\mathrm{D}$ is plotted in Fig. 2E, which shows that the mean velocities driven by $0.18 \mathrm{M}$, $0.36 \mathrm{M}$, and 0.72 M PEG were $99.55 \mathrm{~mm} / \mathrm{h}, 197.16 \mathrm{~mm} / \mathrm{h}$, and $394.21 \mathrm{~mm} / \mathrm{h}$, respectively. At $0.36 \mathrm{M}$ PEG concentration, the flow speed was increased and the colonies of microbiome were detached from the differentiated Caco- 2 cells. In contrast, the flow speed was decreased at a $0.09 \mathrm{M}$ PEG concentration, showing that the colonies of microbiome were rapidly grown. On the basis of these results, we optimized a flow speed of $0.18 \mathrm{M}$ PEG solution, which could produce the most stable fluidic culture model in subsequent experiments.

\subsection{Effect of fluidic flow and endothelial cell on differentiation of epithelial cells in gut on-chip}

We first explored whether the shear stress generated by luminal fluidic flow above the epithelium is responsible for induction of the epithelial morphogenesis. To do this, Caco- 2 cells were grown either in a static culture or microfluidic culture condition (Fig. 3). After 5 days of cultivation, the Caco- 2 cells remained viable in the both fluidic and static conditioned gut-on-a-chip, which was higher cellular density than in the static conditions (Fig. 3A, D). We analyzed the fluidic flow effect on epithelial barrier integrity in Caco- 2 cells confirmed by the immunostaining of junctional ZO-1 and the labeling of the actin cytoskeleton, F-actin. When cultured in a fluidic condition, the actin cytoskeleton showed a continuous ring appearance between adjacent cells, whereas the actin staining appeared the discontinuous and less ordered in a static condition (Fig. 3B, E). In addition, the immunostaining data showed the bright signals of the tight junction protein ( $\mathrm{ZO}-1$ ) at the edge of cells cultured in the fluidic culture condition, suggesting that the Caco- 2 cells could form the confluent polygonal epithelial monolayers with well-developed tight junctions in a fluidic culture condition, much tighter than cells in a static culture condition. (Fig. 3C, F). Nevertheless, the cultured human intestinal epithelial cell alone spontaneously was not formed highly polarized epithelium and mucus secretion cells. To more effectively ameliorates the mimic in vivo intestinal system and differentiation of the Caco-2 cells, we co-cultured Caco- 2 and HUVECs in a gut-on-a chip which separated with $2 \%$ collagen gels (Fig. 4 ). In the same flow conditions, one of the most noticeable changes in a co-culture model, the expression of polarized and differentiated columnar epithelium (Fig. 4A down panel, $\mathrm{F}$-actin) appeared similar form to living in vivo intestinal 


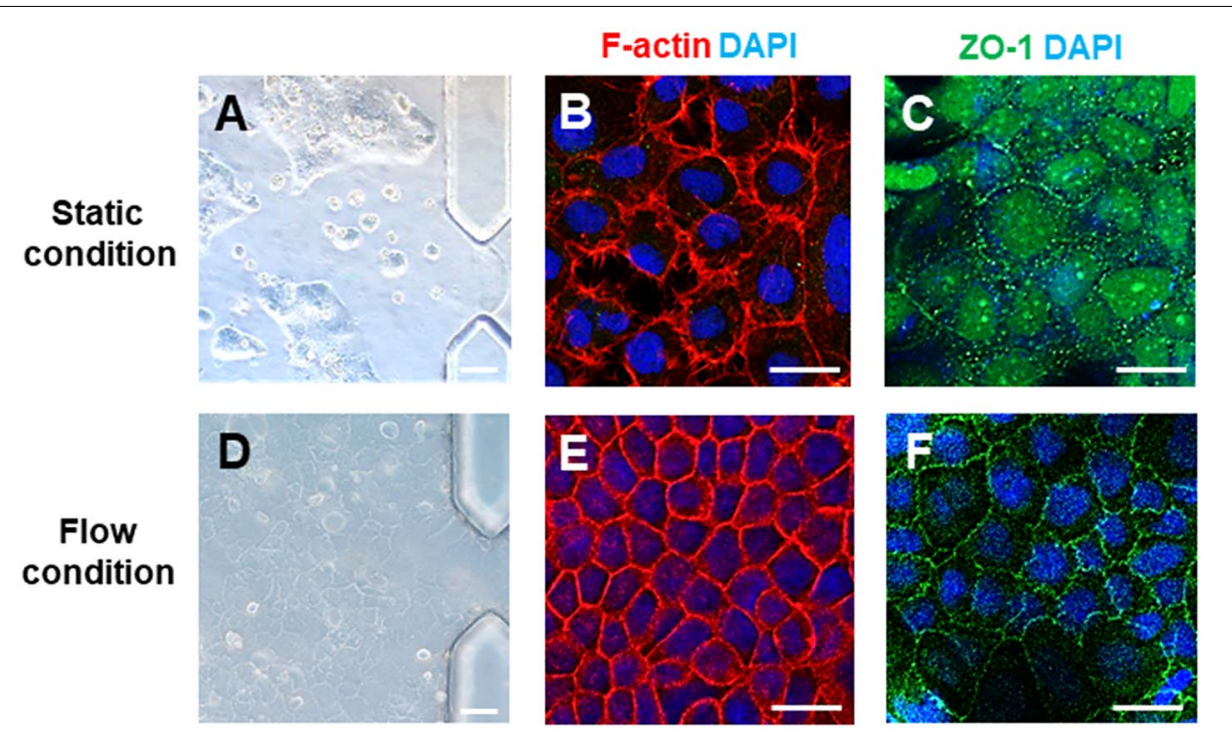

Fig. 3 Morphology of the Caco-2 epithelial cells cultured in a static culture condition and in the gut-on-a-chip with microfluidic flow for 5 days. $\mathbf{A}$ and $\mathbf{D}$ are phase contrast views of Caco-2 cells monolayer. $\mathbf{B}$ and $\mathbf{E}$ show the distribution of the cytoskeleton protein, F-actin, in the Caco-2 cells monolayers. $\mathbf{C}$ and $\mathbf{F}$ show the distribution of the tight junction protein, ZO-1, in the Caco-2 cells monolayers. Scale bars are $25 \mu \mathrm{m}$

villi, as previously described [38]. To investigate the effect of fluidic flow on the differentiation, the 3D projections in the Z-stack of the confocal images were used for quantifying the epithelial layer height (Fig. 4B). The epithelium layer differentiated by Caco- 2 cells cultured in a gut-on-a-chip showed the $37.04 \pm 2.38 \mu \mathrm{m}$ height of the villi, while those under static conditions were only $14.11 \pm 0.74 \mu \mathrm{m}$, respectively (Fig. 4C). Another important characteristic to consider when developing in vitro models of the gut is the presence of a mucus layer, which is most abundant structural protein of the gastrointestinal mucus layer [39]. We evaluated Mucin 2 (MUC2) visualized via immunofluorescence (Fig. 4A down panel, MUC2). We confirmed that the Caco-2 cells cultured in a fluidic culture condition generally produced more MUC2 expression as compared to cells grown in a static culture condition. They were located at the tips of the villi-like structures. Mucin are known to protect the underlying epithelium from mechanical stresses [40], which is expected to be highest at the tip of the villi-like structures under fluidic culture conditions. These results are consistent with previous studies combining Caco-2 and other gastrointestinal cell lines with fluidic flow that reported improved mucus production in response to mechanical stimulation [31, 39]. The Caco-2 cells acted as absorptive enterocytes [41, 42], and formed a continuous, planar epithelial monolayer in transwell inserts after 3 weeks of culture, as previously described [14]. However, they could not exhibit similar in vivo intestinal cell differentiation when grown under static culture conditions. In contrast, in our gut-on-a-chip system, the Caco-2 cell monolayer co-cultured with HUVECs spontaneously initiated villus morphogenesis within 5 days when cultured in the presence of fluidic flow and other supporting cells mimicking the physical microenvironment experienced by in vivo intestinal system. Glycocalyx, an efficient defense system for protecting the epithelium from pathogens [43], was evaluated by expression of WGA-Alexa488, which could bind to sugar residues on cellular surface. A marked increasing in WGA-Alexa488 binding was observed in a flow culture condition as compared to a static condition, suggesting that the flow culture condition could improve the epithelial cell differentiation. As expected, the expression of glycocalyx was influenced by a fluidic condition, indicating that flow condition promoted the differentiation of Caco- 2 cells. Thus, our results suggest that the mechanical factors of the fluidic flow and cellular components are the crucial microenvironment cues that can drive more complete intestinal differentiation process than local stromal factors [44]. Furthermore, these results indicated that Caco- 2 cells cultured in the presence of continuous flow required a shorter time to polarize and differentiate.

\subsection{Effect of fluid flow and endothelial cell on barrier integrity of epithelial cells in gut on-a-chip}

Caco- 2 cell only cultured model and co-cultured model were exposed to fluidic flow for 4 days in a gut-on-achip and the differentiation process was evaluated using impedance spectrometry (Fig. 5). The impedance spectra 


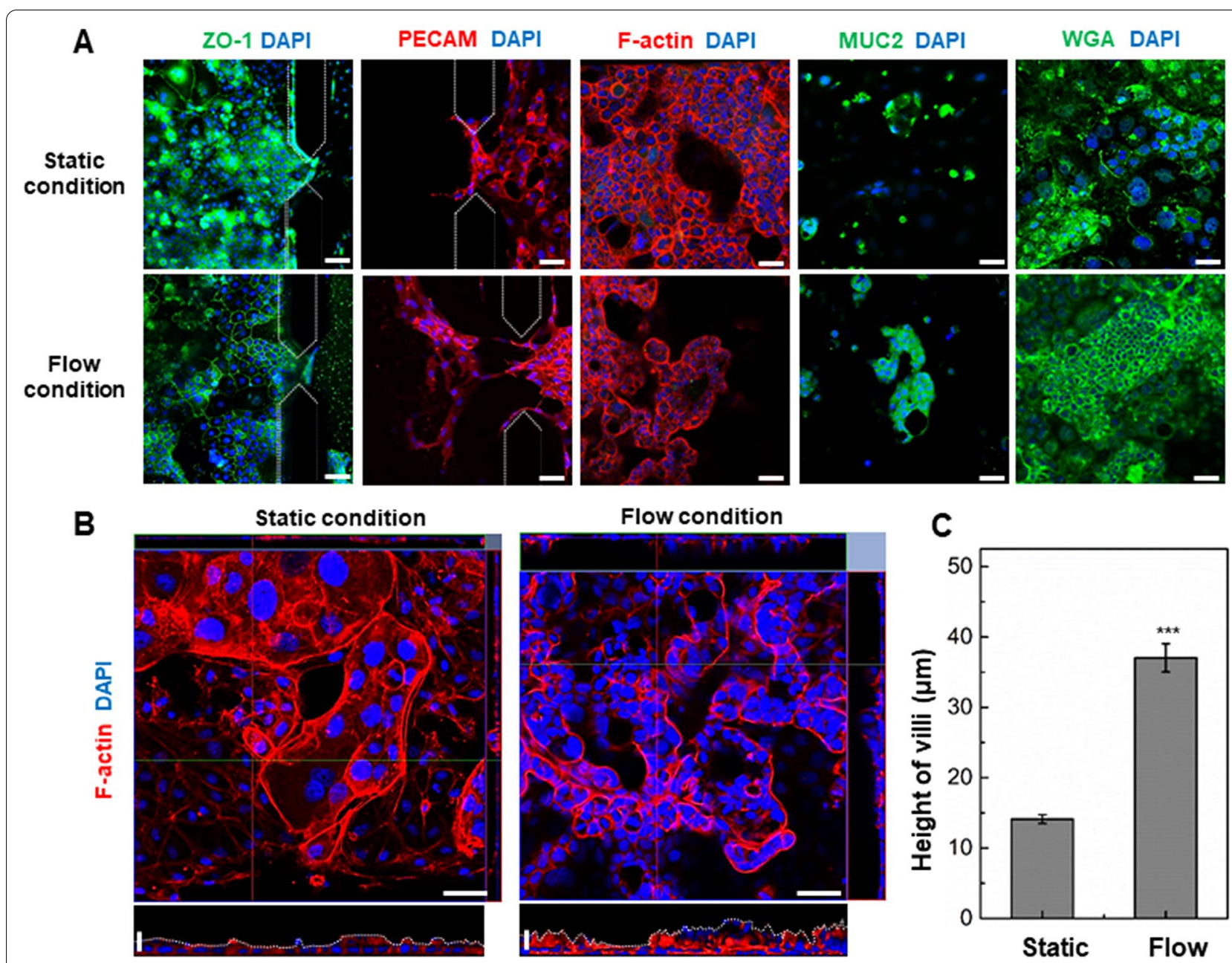

Fig. 4 Morphology of the Caco-2 epithelial cells co-cultured with HUVECs in a static and flow culture condition for 5 days. A Morphological analysis of intestinal specific proteins under a static and flow condition. Scale bars are $50 \mu \mathrm{m}$. B Morphological analysis of polarized columnar epithelium. Fluorescence confocal micrographs (vertical cross-sectional views at 5 days after onset) show a vertical cross-section of the epithelium highlighting cell shape and polarity. Horizontal scale bars are $50 \mu \mathrm{m}$ and vertical scale bars are $25 \mu \mathrm{m}$. C The average height of Caco-2 cells grown in the gut-on-a-chip without and with fluidic flow $\left.{ }^{* * *} p<0.001\right)$

of the Caco-2 cell only cultured model and co-cultured model in a gut-on-a-chip showed that the maximum difference in the impedance spectra was observed at $10 \mathrm{kHz}$ (Fig. 5A, B). The measurement of day 0 before cell seeding was subtracted from all subsequent measurements (resulting in the $\left|Z_{\text {relative }}\right|$ ) to confirm the change in impedance attributed to the cell layer. The relative impedance $\left|\mathrm{Z}_{\text {relative }}\right|$ at $10 \mathrm{kHz}$ was monitored in a Caco-2 cell only cultured model and co-cultured model on a gut-on-a-chip over 4 days (Fig. $5 \mathrm{C}$ ). This non-invasive method can be applied to living cells and allows them to be monitored during growth and differentiation, since their morphological changes can be described by variations in impedance measurements [45]. In particular, it is important for monitoring the growth of cellular extrusions like microvilli [46]. In a gut-on-a-chip with Caco- 2 cell only cultured model and co-cultured model, the measured impedance kept increasing during all 4 days, showing that the Caco- 2 cell only cultured model and co-cultured model was $13 \mathrm{k} \Omega$ and $16 \mathrm{k} \Omega$, respectively. The shear stress induced by the fluidic flow has the effect of the mechanotransduction on several endothelial molecular pathways through activation of membranebound receptors, leading to the production of the tight junction proteins (e.g., ZO-1). It modulated the cytoskeletal structure to promote the cell reorientation and restructuring $[47,48]$. Hereby, the resistance of the sensor surface covered by the cells tends to be increased due to cell proliferation and spreading [49]. Since the current has to flow through the cells, the resistances between the 


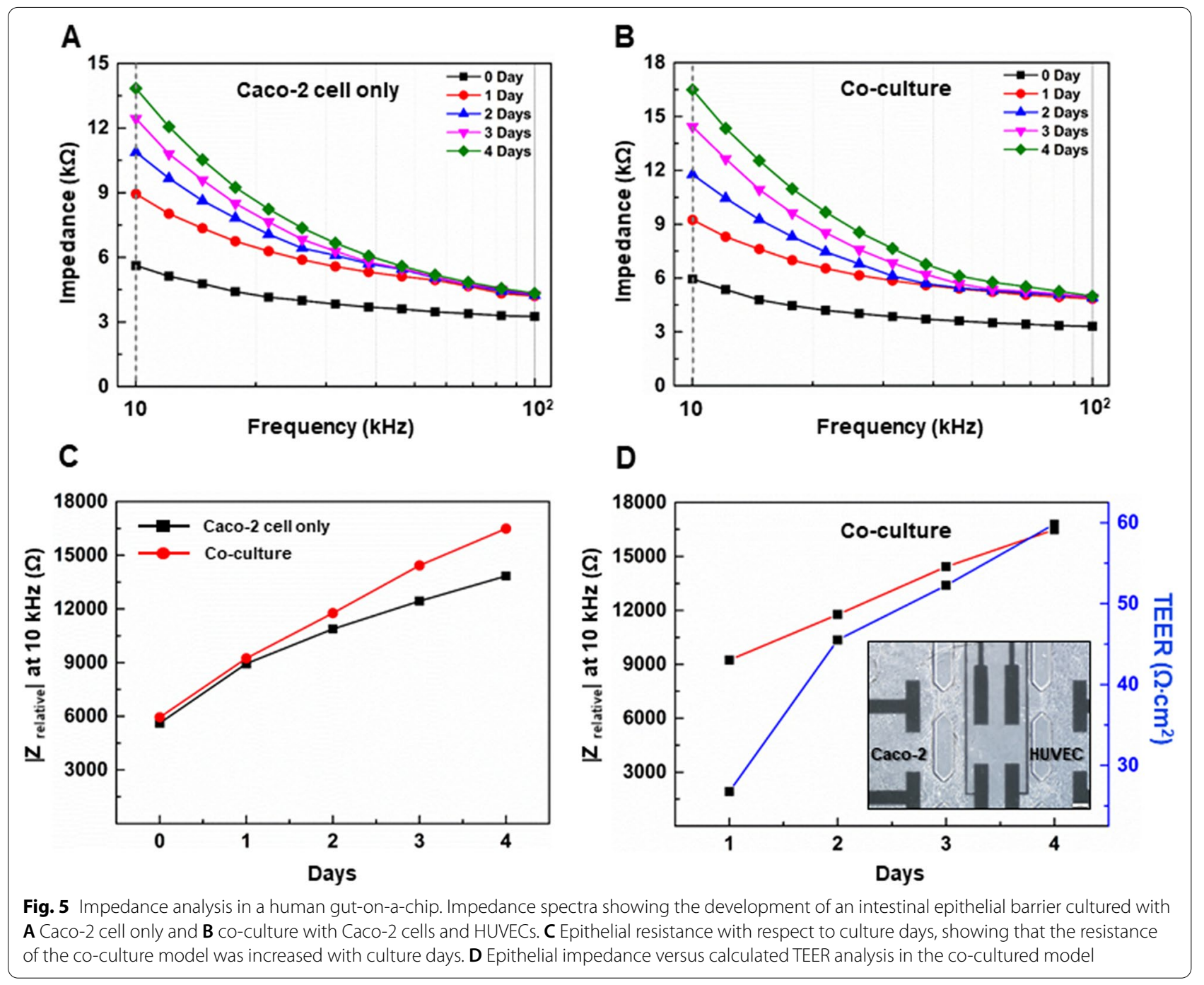

measuring electrodes can keep increasing and the TEER barrier resistance can keep increasing [49]. The relative impedance $|Z|$ and TEER were characterized by an increase in measured cell layer resistance for co-cultured model on a gut-on-a-chip (Fig. 5D). The calculated TEER value was reached up to $59 \Omega \cdot \mathrm{cm}^{2}$ over 4 days of culture. The previous study has reported a time-dependent barrier formation of human intestinal cells developing from 20 to $60 \Omega \cdot \mathrm{cm}^{2}$ in a static culture [50]. This value was comparable to our TEER-measurements. However, our co-cultured model on a gut-on-a-chip showed increase in the barrier strength within 5 days of fluidic flow culture. These results demonstrated that the application of fluidic shear stress and endothelial cells enhanced the cellular differentiation and barrier formation of the intestinal cells.

\subsection{Host cell and probiotic co-culture on gut-on-a-chip} The adhesion of commensal bacteria to host cells is considered as an appropriate parameter to determine the colonization potential of a probiotic strain [51]. However, as the bacterial overgrowth occurs rapidly compromising the epithelium, it is impossible to expose these cells to living microbiome in long-term culture [30]. Thus, the establishment of the stable symbiosis between the epithelium and resident gut microbiome as observed in the normal intestine is crucial to maintain the normal epithelial differentiation and restrain microbial overgrowth in the intestine in vivo [52]. In a present study, we leveraged our gut-on-a-chip to maintain the probiotics. In this study, a commercial probiotic, Lactiplantibacillus plantarum was used as a control, Lactiplantibacillus plantarum HY7715 probiotic and Bifidobacterium animalis spp. lactis HY8002 probiotic 
were employed. Blocking the fluidic flow for the first $2 \mathrm{~h}$, bacterial cells were allowed to adhere on the apical surface of villi. After $2 \mathrm{~h}$, the physiological relevant flow was resumed through the microchannels to remove un-colonized gut bacteria and supply nutrients to both bacterial and villus epithelial cells. When a non-pathogenic laboratory strain of green fluorescent stained probiotics was allowed to adhere to the apical (luminal) surface of villi for $2 \mathrm{~h}$ under static conditions, these bacteria cells were subsequently colonized and spontaneously inhabited regions (Fig. 6). When the bacteria were cultured on the villus epithelium layer under a flow condition ( $21 \mu \mathrm{L} /$ hour $)$, we observed the colonized stable form until day 1 in all probiotic groups. However, Lpb. plantarum and HY8002 probiotic seemed detached from the villi and washed out after 3 days when cultured under flow conditions as compared to HY7715 probiotic, although the luminal flow was maintained constant. All species showed adhesion to the used epithelial layer, however, the adhesion level of
HY7715 probiotic was greater to epithelial layer even in day 5. These results were concurred with the finding by Schillinger et al., who showed that the adherence of diverse probiotic strains varied among strains [53]. In addition, Gopal et al., has reported the higher affinity of L. acidophilus and L. rhamnosus strains to HT29-MTX cells than HT29 and Caco-2 cells [54]. Nevertheless, we need to optimize the incubation time and volumetric flow rate for attachment to the surface of villi.

\subsection{LPS-induced intestinal damage responses and evaluation of barrier protection effect of probiotics}

We further explored whether our gut-on-a-chip system could be used to mimic the human intestinal inflammation in vitro. Following the establishment of the physiologically relevant gut-on-a-chip, we evaluated whether our model could recapitulate the main characteristics of intestinal inflammation. We focused on LPS as alternative stimulus and chose to directly expose the 5 days

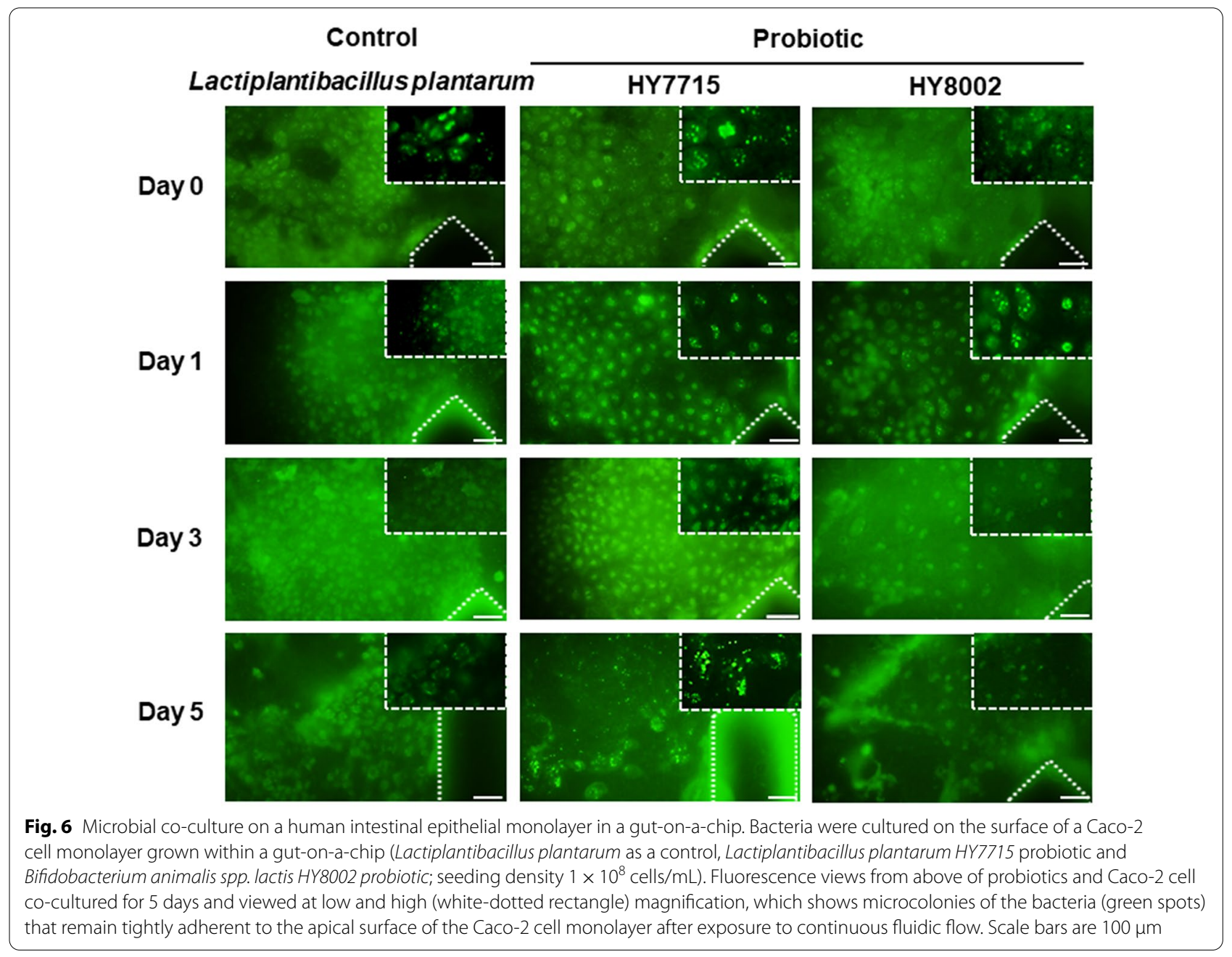


cultured gut-on-a-chip model to induce an inflammation-like response for $24 \mathrm{~h}$. LPS, is a heat-stable toxin associated with the outer membranes of gram-negative bacteria, belongs to the most studied pathogenassociated molecular patterns and generally presents in the intestinal lumen known for its involvement in intestinal inflammation [55]. In a co-cultured model on a gut-on-a-chip exposed to LPS, we observed more disorganized structure and fainter staining of F-actin structures as compared to non-LPS treated models (Fig. 7A, F-actin, white arrows). The location and distribution of the ZO-1 protein was determined by immunofluorescence assay. Under normal conditions, ZO-1 proteins were localized at the cell membrane and appeared as a continuous band encircling the cells at the cellular borders (Fig. 4.) LPS $(15 \mu \mathrm{g} / \mathrm{mL})$ disturbed the distribution of $\mathrm{ZO}-1$ proteins at the cellular borders. LPS also induced obvious cytoplasmic accumulation of ZO-1 in Caco-2 cells (Fig. 7A, ZO-1 staining).
We measured villus heights after LPS administration, because the villus contraction was typically utilized as a measure of small intestinal damage. In co-cultured intestinal models on a gut-on-a-chip at $24 \mathrm{~h}$ after LPS administration, the mean villus height was reduced by $32.9 \%$, indicating $24.84 \pm 0.73 \mu \mathrm{m}$ as compared to villi from non-treated LPS co-cultured intestinal models $\left(37.04 \pm 2.38 \mu \mathrm{m},{ }^{* * * *} p<0.001\right)$ (Fig. 7B). Furthermore, the paracellular permeability was measured by TEER analysis. Administration of $15 \mu \mathrm{g} / \mathrm{mL}$ LPS resulted in a significant decrease in TEER (non-treated LPS and LPS was $59.83 \pm 1.68$ and $28.44 \pm 1.96 \Omega \cdot \mathrm{cm}^{2}$, respectively, $* p<0.05$, Fig. $7 \mathrm{C}$ ). We further evaluated the protective effect of probiotics HY7715 probiotic on LPS-induced epithelial barrier dysfunction. As seen in Fig. 7C, when differentiated Caco-2 cell barriers were treated with $1 \times 10^{8} \mathrm{CFU} / \mathrm{mL}$, the TEER analysis was significantly increased as compared to HY7715 probiotic-treated group after LPS treatment at day 8 (LPS and HY7715
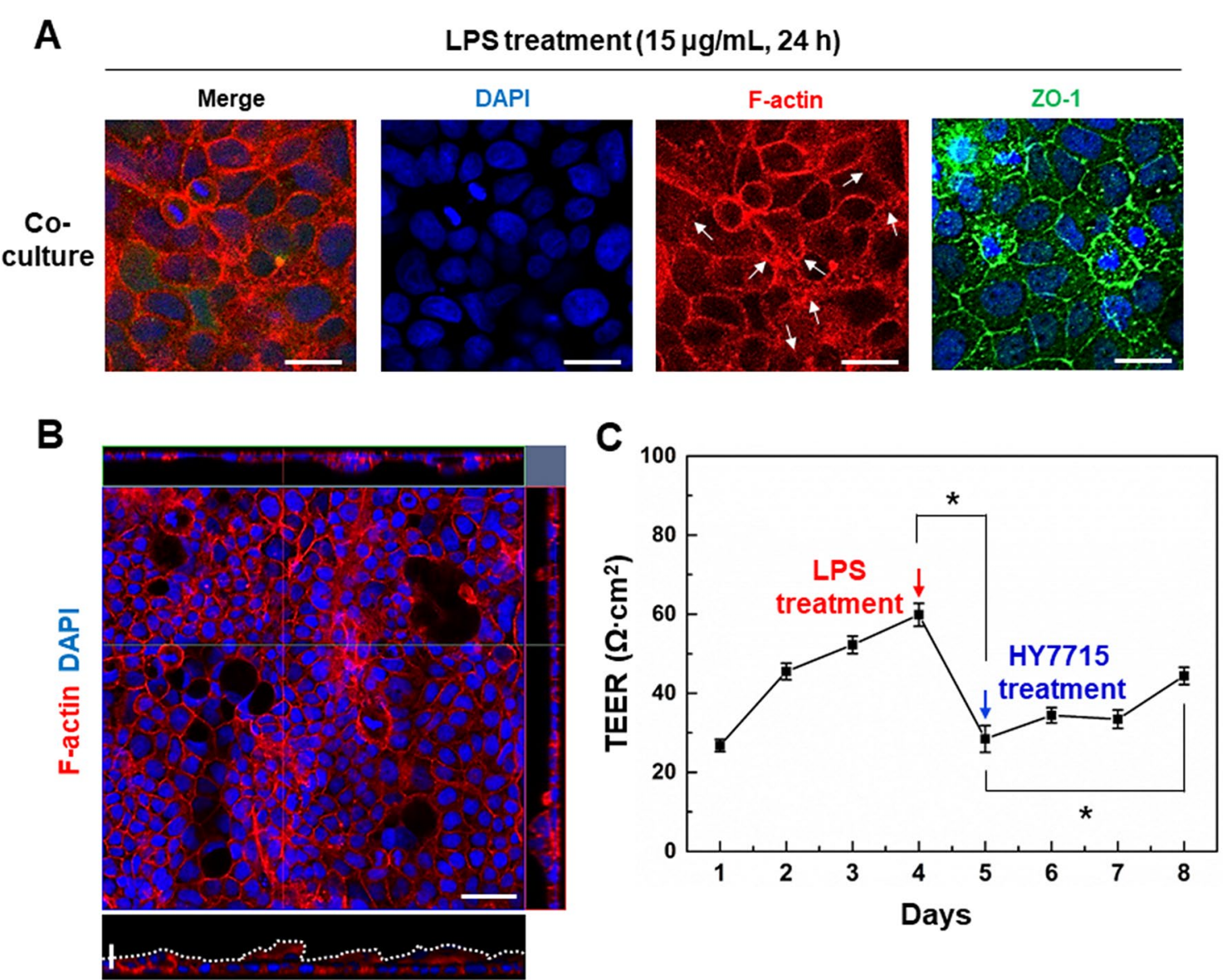

Fig. 7 Induction of pathological intestinal injury induced by non-pathogenic LPS endotoxin and anti-inflammatory effect evaluation of probiotics. A Morphological analysis of the intestinal barrier damage in response to LPS addition. Scale bars are $25 \mu \mathrm{m}$. B Fluorescence confocal micrographs (vertical cross-sectional views) of villi recorded at 5 days after staining with F-actin. Horizontal scale bar is $50 \mu \mathrm{m}$ and Vertical scale bar is $25 \mu \mathrm{m}$. $\mathbf{C}$ Quantification of the intestinal barrier function evaluated by calculated TEER analysis $\left({ }^{*} p<0.05\right)$ 
probiotic was $28.44 \pm 1.96$ and $44.39 \pm 1.25 \Omega \cdot \mathrm{cm}^{2}$, respectively, " $p<0.05)$. These results suggested that the epithelium layer differentiated from Caco-2 cells was damaged by LPS treatment in the co-cultured model on a gut-on-a-chip. Its similar trends were observed in other in vitro gut inflammation models [56] and inflammatory bowel disease (IBD) patients [57]. Additionally, the strain HY7715 probiotic suppressed LPS-induced decreases in TEER analysis on a co-cultured intestinal models. It suggests that strain HY7715 probiotic can play an important role in changes in intestinal cell permeability. This result is consistent with the finding that probiotic strains of bacteria, including Lactobacillus rhamnosus GG (LGG), have been reported to elevate intestinal epithelial integrity [58] in vitro and improve intestinal barrier function in a human [59]. The previous studies have reported that L. plantarum significantly reduced the production of inflammatory cytokines and gut permeability in an IBD pathology by regulating the LPS [60]. This result demonstrated that intestinal epithelial integrity significantly increased in the presence of HY7715 probiotic co-cultures. The presence of the probiotics clearly provides useful microenvironmental signals that enhance epithelial cell functions, which are necessary to maintain this dynamic interface.

\section{Conclusions}

The human microbiomeplays a crucial role in treating the intestinal health and disease. In our gut-on-a-chip system with microelectrode arrays, we cultured the human epithelial cells in a left channel and endothelial cells in a right channel, allowing to simulate and study the impact of fluidic flow and endothelial cells on epithelial cell differentiation without the influence of physical cell contact. Additionally, our gut-on-achip co-culture system enables to the perfusion-based cell culture with microbiomes and analyzes the effect of microbiomes to intestinal epithelial barrier functions in vitro. Therefore, our human intestinal gut-ona-chip system to investigate host-microbe interaction could be a potentially powerful tool for pharmaceutical applications.

\section{Abbreviations}

IBD: Inflammatory bowel disease; Caco-2: Human intestinal epithelial cells; LPS: Lipopolysaccharide LPS; PDMS: Polydimethylsiloxane; HUVECs: Human umbilical vein endothelial cells; TEER: Transepithelial electrical resistance; MUC2: Mucin 2.

\section{Acknowledgements}

Not applicable.

\section{Authors' contributions}

M.S.J. and Y.Y.C. designed the experiments and analyzed the data as well as they are equally contributed to this work. S.J.M., J.H.H., Y.S.L., and H.U.L. analyzed the probiotic, TEER, and simulation data. S.D.P., J.-.S.S., J.-L.L. performed the project administration. B.G. Chung supervised the project and analyzed the data. All authors read and approved the final manuscript.

\section{Funding}

This work was supported by hy Co., Ltd. in Korea (Grant Number 2021-00048).

\section{Availability of data and materials}

The authors have no data to share since all data are shown in the submitted manuscript.

\section{Declarations}

\section{Ethics approval and consent to participate}

Not applicable.

\section{Competing interests}

The authors declare that they have no known competing financial interests or personal relationships that could have appeared to influence the work reported in this paper.

\section{Author details}

'Department of Biomedical Engineering, Sogang University, Seoul, Korea. ${ }^{2}$ Institute of Integrated Biotechnology, Sogang University, Seoul, Korea. ${ }^{3}$ R\&BD Center, hy Co., Ltd., Yongin-si, Korea. ${ }^{4}$ Department of Mechanical Engineering, Sogang University, Seoul, Korea.

Received: 10 January 2022 Accepted: 18 January 2022

Published online: 08 February 2022 
28. D. Huh, B.D. Matthews, A. Mammoto, M. Montoya-Zavala, H.Y. Hsin, D.E. Ingber, Science 328, 1662 (2010)

29. C.L. Maynard, C.O. Elson, R.D. Hatton, C.T. Weaver, Nature 489, 231 (2012)

30. H.J. Kim, D. Huh, G. Hamilton, D.E. Ingber, Lab Chip 12, 2165 (2012)

31. H.J. Kim, D.E. Ingber, Integr Biol 5, 1130 (2013)

32. J.Y. Park, C.M. Hwang, S.H. Lee, S.H. Lee, Lab Chip 7, 1673 (2007)

33. J. Haorah, K. Schall, S.H. Ramirez, Y. Persidsky, Glia 56, 78 (2008)

34. P.M. Watson, J.C. Paterson, G. Thom, U. Ginman, S. Lundquist, C.I. Webster, BMC Neurosci 14, 59 (2013)

35. M.W. van der Helm, M. Odijk, J.P. Frimat, A.D. van der Meer, J.C.T. Eijkel, A. van den Berg, L.I. Segerink, Biosens Bioelectron 85, 924 (2016)

36. K.M. Ainslie, J.S. Garanich, R.O. Dull, J.M. Tarbell, J Appl Physiol (1985) 98, 242 (2005)

37. H. Haegland, H.K. Dahle, G.T. Eigestad, K.A. Lie, I. Aavatsmark, Adv Water Resour 30, 1027 (2007)

38. P.G. Toner, K.E. Carr, J Pathol 97, 611 (1969)

39. N. Navabi, M.A. McGuckin, S.K. Linden, PLoS ONE (2013). https://doi.org/ 10.1371/journal.pone.0068761

40. M.E. Johansson, H. Sjovall, G.C. Hansson, Nat Rev Gastroenterol Hepatol 10, 352 (2013)

41. Q.M. Ding, T.C. Ko, B.M. Evers, Am J Physiol 275, C1193 (1998)

42. L.P. Pageot, N. Perreault, N. Basora, C. Francoeur, P. Magny, J.F. Beaulieu, Microsc Res Tech 49, 394 (2000)

43. J. Liu, B. Williams, D. Frank, S.M. Dillon, C.C. Wilson, A.L. Landay, J Immunol 198, 605 (2017)

44. V. Meunier, M. Bourrie, Y. Berger, G. Fabre, Cell Biol Toxicol 11, 187 (1995)

45. M. Marziano et al., Biochim Biophys Acta Gen Subj 1863, 893 (2019)

46. K. Benson, S. Cramer, H.J. Galla, Fluids Barriers CNS 10, 5 (2013)

47. V. Siddharthan, Y.V. Kim, S. Liu, K.S. Kim, Brain Res 1147, 39 (2007)

48. C.G. Galbraith, R. Skalak, S. Chien, Cell Motil Cytoskeleton 40, 317 (1998)

49. E. Bossink, M. Zakharova, D.S. de Bruijn, M. Odijk, L.I. Segerink, Lab Chip 21, 2040 (2021)

50. A. Beduneau, C. Tempesta, S. Fimbel, Y. Pellequer, V. Jannin, F. Demarne, A. Lamprecht, Eur J Pharm Biopharm 87, 290 (2014)

51. A.L. Servin, M.H. Coconnier, Best Pract Res Clin Gastroenterol 17, 741 (2003)

52. G. Vantrappen, J. Janssens, J. Hellemans, Y. Ghoos, J Clin Invest 59, 1158 (1977)

53. U. Schillinger, C. Guigas, W.H. Holzapfel, Int Dairy J 15, 1289 (2005)

54. P.K. Gopal, J. Prasad, J. Smart, H.S. Gill, Int J Food Microbiol 67, 207 (2001)

55. S. Guo, R. Al-Sadi, H.M. Said, T.Y. Ma, Am J Pathol 182, 375 (2013)

56. J.J. Park, H.J. Moon, J.H. Park, T.H. Kwon, Y.K. Park, J.H. Kim, J Neurosurg Spine 24, 167 (2016)

57. P. Suenaert, V. Bulteel, L. Lemmens, M. Noman, B. Geypens, G. Van Assche, K. Geboes, J.L. Ceuppens, P. Rutgeerts, Am J Gastroenterol 97, 2000 (2002)

58. H.W. Fang, S.B. Fang, J.S. Chiang Chiau, C.Y. Yeung, W.T. Chan, C.B. Jiang, M.L. Cheng, H.C. Lee, J Med Microbiol 59, 573 (2010)

59. C. Dai, D.H. Zhao, M. Jiang, Int J Mol Med 29, 202 (2012)

60. C. Chelakkot, J. Ghim, S.H. Ryu, Exp Mol Med 50, 1 (2018)

\section{Publisher's Note}

Springer Nature remains neutral with regard to jurisdictional claims in published maps and institutional affiliations.

\section{Submit your manuscript to a SpringerOpen ${ }^{\circ}$ journal and benefit from:}

- Convenient online submission

- Rigorous peer review

- Open access: articles freely available online

- High visibility within the field

- Retaining the copyright to your article

Submit your next manuscript at $\boldsymbol{\nabla}$ springeropen.com 